\title{
Converting Interpolation Series into Chebyshev Series by Recurrence Formulas
}

\author{
By Herbert E. Salzer
}

\begin{abstract}
Interpolation series (divided difference, Gregory-Newton, Gauss, Stirling, Bessel) are converted into Chebyshev (or Jacobi) series by applying a previously derived general five-term recurrence formula [3]. It employs the coefficients in three-term linear recurrence formulas (same kind as for orthogonal polynomials) which have been found for the $m$ th degree nonorthogonal polynomial coefficients of the differences used in the interpolation series. In the Gauss, Stirling and Bessel series, the coefficients in the recurrence formulas vary with the parity of $m$. The basic five-term recurrence formula is applicable also to: (1) inter- and intraconversion of power series in $a x+b$, divided difference and equal-interval interpolation series (including subtabulation), and Chebyshev series, (2) obtaining Chebyshev series for solutions of difference equations, (3) the derivation of formulas for Chebyshev coefficients in terms of differences, and (4) the conversion of interpolation series into Chebyshev series, for more than one variable.
\end{abstract}

Introduction. In a previous note on converting from one orthogonal polynomial series $\Sigma_{m=0}^{n} a_{m} q_{m}(x)$ into another orthogonal polynomial series $\Sigma_{m=0}^{n} A_{m} Q_{m}(x)$ [3] , the basic five-term recurrence formula $[3,(22)]$ is deduced just from these three-term recurrence formulas for $q_{m} \equiv q_{m}(x)$ and $Q_{m} \equiv Q_{m}(x)$ :

$$
q_{-1}=0, \quad q_{m+1}+(a(m)+b(m) x) q_{m}+c(m) q_{m-1}=0,
$$

$$
Q_{-1}=0, \quad Q_{m+1}+(A(m)+B(m) x) Q_{m}+C(m) Q_{m-1}=0, \quad m=0(1) n-1 .
$$

Since the orthogonality of $q_{m}$ or $Q_{m}$ is not necessary for (1a) or (1b) respectively, it happens that $[3,(22)]$ has many applications when either of, or both, $q_{m}$ and $Q_{m}$ are not orthogonal. We give here some useful applications to converting a number of different interpolation series into Chebyshev series. These seven interpolation series, namely, Newton's divided difference formula, the Gregory-Newton formulas with forward and backward differences, Gauss's forward and backward formulas, Stirling's formula and Bessel's formula [2], through the $n$th degree terms, ${ }^{1}$ are each expressible as $\sum_{m=0}^{n} a_{m} q_{m}$ where $q_{m}$ satisfies a three-term recurrence relation of the form (1a). This

Received June 16, 1975.

AMS (MOS) subject classifications (1970). Primary 65D05, 33A65, 40-04; Secondary 41 A05, $41 \mathrm{~A} 10,41 \mathrm{~A} 30$.

Key words and phrases. Series interconversion, interpolation series, Chebyshev series, recurrence formulas, three-term recurrence, five-term recurrence, difference equations.

1 Remainder terms are not considered here. 
is not so obvious in the Gauss, Stirling and Bessel formulas, especially since $a(m), b(m)$ and $c(m)$ in (1a) depend also upon the parity of $m$.

Notation. We retain here the same notations as in [3], except for the variable $p$ in place of the variable $x$, where $p=\left(x-x_{0}\right) / h$ for the tabular interval $h$, in all the equal-interval interpolation formulas (i.e., in all but the divided difference formula). We express all interpolation formulas in a notation that is slightly different from that in [2] and also somewhat more compact, but which expresses the $q_{m}$ in $P_{n}(x)$ or $P_{n}\left(x_{0}+p h\right)=\Sigma_{m=0}^{n} a_{m} q_{m}$ directly in terms of $m$ and $x$ or $m$ and $p$, respectively. All differences or divided differences, as well as Chebyshev or other coefficients, are those of the $n$th degree $(n+1)$-point interpolation polynomial $P_{n}(x)$ which is not the same as $f(x)$, the function that is tabulated, when $f(x)$ is not an exact $n$th degree polynomial. ${ }^{2}$ Thus, here $D_{x}^{m}$ denotes the $m$ th divided difference of $P_{n}(x)$ for $\left(x_{0}, x_{1}, \ldots, x_{m}\right)$. Other difference notations are standard, such as $\Delta_{0}^{m}$ or $\nabla_{0}^{m}$ for the $m$ th forward or backward difference of $P_{n}\left(x_{0}+p h\right)$ at $x=x_{0}$, and $\delta_{0}^{m}$ or $\mu \delta_{0}^{m}$ and $\delta_{1 / 2}^{m}$ or $\mu \delta_{1 / 2}^{m}$ for $m$ th central or mean central differences of $P_{n}\left(x_{0}+p h\right)$ at $x_{0}$ and $x_{0}+1 / 2 h$, respectively.

Recurrence Scheme for Conversion of Series. To make this note self-sufficient, the previously published general five-term recurrence scheme [3, (22)], for obtaining $A_{m}=a_{m}^{(n)}$ from $a_{m}, m=O(1) n$, is given here in this more condensed form:

$$
a_{m}^{(k+1)}=-a_{m}^{(k-1)} c(n-k)+a_{m-1}^{(k)} b(n-k-1) / B(m-1)
$$

$$
\begin{aligned}
& +a_{m}^{(k)}[-a(n-k-1)+b(n-k-1) A(m) / B(m)] \\
& +a_{m+1}^{(k)} b(n-k-1) C(m+1) / B(m+1)+\delta_{m}^{0} a_{n-k-1}, \\
& m=0(1) k+1, \quad k=-1(1) n-1,
\end{aligned}
$$

where $a_{i}^{(j)}=0$ for $i<0$ or $i>j$, and $\delta_{m}^{0}=0, m \neq 0, \delta_{m}^{0}=1, m=0$.

Interpolation Polynomials and Recurrence Coefficients. Following are the seven above-mentioned interpolation polynomials, each written in the form $\sum_{m=0}^{n} a_{m} q_{m}(x$ or $p),{ }^{3}$ together with the corresponding coefficients $a(m), b(m)$ and $c(m)$ in the recurrence formula (1a). In every case $a_{0}=P_{n}\left(x_{0}\right)=f\left(x_{0}\right)=\Delta_{0}^{0}=\nabla_{0}^{0}=\delta_{0}^{0}, q_{0}=1$.

I. Newton Divided Difference Formula.

$$
P_{n}(x)=P_{n}\left(x_{0}\right)+\sum_{m=1}^{n}\left[\prod_{i=0}^{m-1}\left(x-x_{i}\right)\right] D_{x}^{m},
$$

$$
a_{m}=D_{x}^{m}, \quad q_{m}=\prod_{i=0}^{m-1}\left(x-x_{i}\right), \quad m=1(1) n
$$

2 Even though initially $P_{n}(x)$ must have the identical $n$ differences of $f(x)$ upon which $P_{n}(x)$ is constructed, the point here being that all subsequent series conversions are for $P_{n}(x)$, and not $f(x)$.

3 Strictly speaking, the form is $\Sigma_{m=0}^{n} q_{m}(x$ or $p) a_{m}$ since, by custom, the differences $a_{m}$ are written after the $m$ th degree polynomial coefficients $q_{m}(x$ or $p)$. 


$$
a(m)=x_{m}, \quad b(m)=-1, \quad c(m)=0, \quad m=0(1) n-1 .
$$

IIa. Newton Forward Difference Formula.

$$
\begin{aligned}
& P_{n}\left(x_{0}+p h\right)=P_{n}\left(x_{0}\right)+\sum_{m=1}^{n}\left(\begin{array}{c}
p \\
m
\end{array}\right) \Delta_{0}^{m}, \\
& a_{m}=\Delta_{0}^{m}, \quad q_{m}=\left(\begin{array}{c}
p \\
m
\end{array}\right), \quad m=0(1) n,
\end{aligned}
$$

(7) $\quad a(m)=m /(m+1), \quad b(m)=-1 /(m+1), \quad c(m)=0, \quad m=0(1) n-1$.

Ilb. Newton Backward Difference Formula.

$$
P_{n}\left(x_{0}+p h\right)=P_{n}\left(x_{0}\right)+\sum_{m=1}^{n}\left(\begin{array}{c}
p+m-1 \\
m
\end{array}\right) \nabla_{0}^{m},
$$

$$
a_{m}=\nabla_{0}^{m}, \quad q_{m}=\left(\begin{array}{c}
p+m-1 \\
m
\end{array}\right), \quad m=0(1) n
$$

(10) $\quad a(m)=-m /(m+1), \quad b(m)=-1 /(m+1), \quad c(m)=0, \quad m=0(1) n-1$.

IIIa. ,Gauss Forward Formula.

$$
\begin{aligned}
P_{n}\left(x_{0}+p h\right)= & P_{n}\left(x_{0}\right)+\left(\begin{array}{c}
p \\
1
\end{array}\right) \delta_{1 / 2}+\left(\begin{array}{c}
p \\
2
\end{array}\right) \delta_{0}^{2} \\
& +\left(\begin{array}{c}
p+1 \\
3
\end{array}\right) \delta_{1 / 2}^{3}+\left(\begin{array}{c}
p+1 \\
4
\end{array}\right) \delta_{0}^{4}+\cdots,
\end{aligned}
$$

$$
a_{m}=\delta_{0}^{m}, \quad q_{m}=\left(\begin{array}{c}
p+m / 2-1 \\
m
\end{array}\right), \quad m \text { even, }{ }^{4}
$$

$$
a_{m}=\delta_{1 / 2}^{m}, \quad q_{m}=\left(\begin{array}{c}
p+(m-1) / 2 \\
m
\end{array}\right), \quad m \text { odd }
$$

(13a) $a(m)=-m / 2(m+1), \quad b(m)=-1 /(m+1), \quad c(m)=0, \quad m$ even,

IIIb. Gauss Backward Formula.

$$
\begin{aligned}
P_{n}\left(x_{0}+p h\right)= & P_{n}\left(x_{0}\right)+\left(\begin{array}{c}
p \\
1
\end{array}\right) \delta_{-1 / 2}+\left(\begin{array}{c}
p+1 \\
2
\end{array}\right) \delta_{0}^{2} \\
& +\left(\begin{array}{c}
p+1 \\
3
\end{array}\right) \delta_{-1 / 2}^{3}+\left(\begin{array}{c}
p+2 \\
4
\end{array}\right) \delta_{0}^{4}+\cdots
\end{aligned}
$$

$$
\begin{array}{cc}
a_{m}=\delta_{0}^{m}, & q_{m}=\left(\begin{array}{c}
p+m / 2 \\
m
\end{array}\right), \quad m \text { even, } \\
a_{m}=\delta_{-1 / 2}^{m}, & q_{m}=\left(\begin{array}{c}
p+(m-1) / 2 \\
m
\end{array}\right), \quad m \text { odd } .
\end{array}
$$

(16a) $a(m)=m / 2(m+1), \quad b(m)=-1 /(m+1), \quad c(m)=0, \quad m$ even, 
(16b)

$$
a(m)=-1 / 2, \quad b(m)=-1 /(m+1), \quad c(m)=0, \quad m \text { odd } .
$$

IV. Stirling Formula.

$$
\begin{aligned}
P_{n}\left(x_{0}+p h\right)= & P_{n}\left(x_{0}\right)+\left(\begin{array}{c}
p \\
1
\end{array}\right) \mu \delta_{0}+\frac{p}{2}\left(\begin{array}{c}
p \\
1
\end{array}\right) \delta_{0}^{2} \\
& +\left(\begin{array}{c}
p+1 \\
3
\end{array}\right) \mu \delta_{0}^{3}+\frac{p}{4}\left(\begin{array}{c}
p+1 \\
3
\end{array}\right) \delta_{0}^{4}+\cdots,
\end{aligned}
$$

$$
a_{m}=\delta_{0}^{m}, \quad q_{m}=\frac{p}{m}\left(\begin{array}{c}
p+m / 2-1 \\
m-1
\end{array}\right), \quad m \text { even }(>0),
$$

$$
a_{m}=\mu \delta_{0}^{m}, \quad q_{m}=\left(\begin{array}{c}
p+(m-1) / 2 \\
m
\end{array}\right), \quad m \text { odd } .
$$

$$
a(m)=0, \quad b(m)=-1 /(m+1), \quad c(m)=m / 4(m+1), \quad m \text { even, }
$$

$$
a(m)=0, \quad b(m)=-1 /(m+1), \quad c(m)=0, \quad m \text { odd } .
$$

V. Bessel Formula.

$$
\begin{aligned}
P_{n}\left(x_{0}+p h\right)= & P_{n}\left(x_{0}\right)+\left(\begin{array}{c}
p \\
1
\end{array}\right) \delta_{1 / 2}+\left(\begin{array}{l}
p \\
2
\end{array}\right) \mu \delta_{1 / 2}^{2}+\frac{p(p-1 / 2)(p-1)}{3 !} \delta_{1 / 2}^{3} \\
& +\left(\begin{array}{c}
p+1 \\
4
\end{array}\right) \mu \delta_{1 / 2}^{4}+\cdots
\end{aligned}
$$

$$
a_{m}=\mu \delta_{1 / 2}^{m}, \quad q_{m}=\left(\begin{array}{c}
p+m / 2-1 \\
m
\end{array}\right), \quad m \text { even }(>0),
$$

(21b) $\quad a_{m}=\delta_{1 / 2}^{m}, \quad q_{m}=\frac{p-1 / 2}{m}\left(\begin{array}{c}p+(m-3) / 2 \\ m-1\end{array}\right), \quad m$ odd $(>1)$.

$$
a(0)=0, \quad b(0)=-1, \quad c(0)=0,
$$

(22a) $a(m)=1 / 2(m+1), \quad b(m)=-1 /(m+1), \quad c(m)=0, \quad m$ even $(>0)$.

$$
a(m)=1 / 2(m+1), \quad b(m)=-1 /(m+1), \quad c(m)=m / 4(m+1),
$$

$m$ odd $(>1)$.

Choice of Interval for Chebyshev Polynomials. To convert the preceding interpolation polynomials into Chebyshev series by $(1 \mathrm{c})$, we need $A(m), B(m)$ and $C(m)$ for the Chebyshev polynomials that have been obtained from $T_{m} \equiv T_{m}(x)=$ $\cos (m \operatorname{arc} \cos x), m \geqslant 0$, after the $[-1,1]$ interval for $x$ (or $p$ ) has been transformed linearly into an interval $[a, b]$ that is best suited to the interpolation formula. For $T_{m}$ itself, when the best interval is $[-1,1]$, we have the recurrence relation

$$
T_{0}=1, \quad T_{1}=x, \quad T_{m+1}=2 x T_{m}-T_{m-1}, \quad m>0,
$$

so that 


$$
\begin{aligned}
& A(0)=C(0)=0, \quad B(0)=-1 ; \quad A(m)=0, \quad B(m)=-2, \quad C(m)=1, \\
& m>0 \text {. }
\end{aligned}
$$

For the interval $[a, b], T_{m}$ is transformed into $T_{m}^{(a, b)} \equiv T_{m}((2 x-a-b) /(b-a))$, which satisfies the recurrence relation

$$
\begin{gathered}
T_{0}^{(a, b)}=1, \quad T_{1}^{(a, b)}=\frac{2 x-a-b}{b-a}, \\
T_{m+1}^{(a, b)}=\frac{4 x-2 a-2 b}{b-a} T_{m}^{(a, b)}-T_{m-1}^{(a, b)}, \quad m>0,
\end{gathered}
$$

so that

$$
\begin{gathered}
A(0)=(b+a) /(b-a), \quad B(0)=-2 /(b-a), \quad C(0)=0 \\
A(m)=\frac{2(b+a)}{b-a}, \quad B(m)=-\frac{4}{b-a}, \quad C(m)=1, \quad m>0 .
\end{gathered}
$$

For $\mathrm{I}$, it is natural to employ $T_{m}^{(a, b)}$ where $[a, b]$ is the smallest interval that includes $x_{i}, i=0(1) n$, and $x$. For IIa and IIb, where $p$ usually lies within $[0,1]$, we convert to a series in $T_{m}^{(0,1)}$, often written as $T_{m}^{*}$, for which

$$
\begin{array}{rlrl}
A(0) & =1, & B(0)=-2, & C(0)=0 ; \\
A(m)=2, & B(m)=-4, & C(m)=1, \quad m>0 .
\end{array}
$$

However, when IIa or IIb is used for both interpolation and extrapolation as far as one tabular interval, in which case $p$ might be anywhere within the interval $[-1,1]$, we convert to a series in $T_{m}^{(-1,1)} \equiv T_{m}$. For IIIa, $p$ is most likely to be in $[0,1 / 2]$ and the Chebyshev series is in terms of $T_{m}^{(0,1 / 2)}$, for which

$$
\begin{array}{llll}
A(0) & =1, & B(0)=-4, & C(0)=0 ; \\
A(m)=2, & B(m)=-8, & C(m)=1, & m>0 .
\end{array}
$$

Similarly for IIIb, where $p$ is within $[-1 / 2,0]$, we transform to $T_{m}^{(-1 / 2,0)}$ for which

$$
\begin{array}{lll}
A(0)=-1, & B(0)=-4, & C(0)=0 ; \\
A(m)=-2, & B(m)=-8, & C(m)=1, \quad m>0 .
\end{array}
$$

For IV, $p$ is most likely to lie within $[-1 / 2,1 / 2]$, and we employ $T_{m}^{(-1 / 2,1 / 2)}$ for which

$$
\begin{aligned}
& A(0)=C(0)=0, \quad B(0)=-2 ; \\
& A(m)=0, \quad B(m)=-4, \quad C(m)=1, \quad m>0 .
\end{aligned}
$$

But should $p$ be confined to either of the intervals $[0,1 / 2]$ or $[-1 / 2,0]$, we employ, of course, $T_{m}^{(0,1 / 2)}$ or $T_{m}^{(-1 / 2,0)}$, respectively. For $\mathrm{V}, p$ is most likely to be within $[0,1]$ for which we transform to $T_{m}^{(0,1)} \equiv T_{m}^{*}$. But if $p$ should happen to be confined to either of the intervals $[0,1 / 2]$ or $[1 / 2,1]$, we should transform to $T_{m}^{(0,1 / 2)}$ or $T_{m}^{(1 / 2,1)}$, respectively, where for $T_{m}^{(1 / 2,1)}$, 


$$
\begin{array}{lll}
A(0)=3, & B(0)=-4, & C(0)=0 ; \\
A(m)=6, & B(m)=-8, & C(m)=1, \quad m>0 .
\end{array}
$$

For some purposes one might wish to convert the interpolation polynomial $P_{n}(x)$ into a series in other than Chebyshev polynomials, such as Jacobi polynomials $P_{m}^{(\alpha, \beta)} \equiv$ $P_{m}^{(\alpha, \beta)}(x)$, for suitable $\alpha$ and $\beta$. For the interval $[-1,1]$ we have

$$
\begin{aligned}
& A(0)=-1 / 2(\alpha-\beta), \quad B(0)=-1 / 2(\alpha+\beta+2), \quad C(0)=0 ; \\
& A(m)=-\left(\alpha^{2}-\beta^{2}\right) \frac{(2 m+\alpha+\beta+1)}{D(m)}, \\
& B(m)=-(2 m+\alpha+\beta)(2 m+\alpha+\beta+1) \frac{(2 m+\alpha+\beta+2)}{D(m)}, \\
& C(m)=2(m+\alpha)(m+\beta) \frac{(2 m+\alpha+\beta+2)}{D(m)}, \\
& D(m)=2(m+1)(m+\alpha+\beta+1)(2 m+\alpha+\beta), \quad m>0 .
\end{aligned}
$$

As in the Chebyshev series above, to obtain a Jacobi series for an interval $[a, b]$ that is specially suited to the interpolation series, we employ $P_{m}^{(\alpha, \beta)}((2 x($ or $2 p)-a-b) /(b-a))$, and for $m \geqslant 0, A(m)$ is replaced by $A(m)-(a+b) B(m) /(b-a), B(m)$ by $2 B(m) /(b-a)$, while $C(m)$ is unchanged. For a Jacobi series giving a good unweighted least-square type of approximation, we might choose $\alpha=\beta=0$, i.e., the Legendre polynomials $P_{m} \equiv P_{m}^{(0,0)}(x)$, where for the interval $[-1,1]$ we have

$$
\begin{aligned}
& A(0)=C(0)=0, \quad B(0)=-1 ; \\
& A(m)=0, \quad B(m)=-\frac{(2 m+1)}{(m+1)}, \quad C(m)=\frac{m}{(m+1)}, \quad m>0,
\end{aligned}
$$

and after transformation to $[a, b]$,

$$
\begin{aligned}
& A(0)=\frac{b+a}{b-a}, \quad B(0)=-\frac{2}{b-a}, \quad C(0)=0 \\
& A(m)=\frac{(2 m+1)(b+a)}{(m+1)(b-a)}, \quad B(m)=-\frac{2(2 m+1)}{(m+1)(b-a)}, \\
& C(m)=\frac{m}{m+1}, \quad m>0 .
\end{aligned}
$$

Other Related Applications of (1c). ${ }^{5} \quad$ It is worth noting that (1c) is also useful for interconversion of power series $\Sigma_{m=0}^{n} a_{m}(a+b x)^{m}$, divided difference series and Chebyshev series, including cases of intraconversion, namely, $\Sigma_{m=0}^{n} a_{m}(a+b x)^{m}$ into $\sum_{m=0}^{n} A_{m}(A+B x)^{m}$,

$$
P_{n}\left(x_{0}\right)+\sum_{m=1}^{n}\left[\prod_{i=0}^{m-1}\left(x-x_{i}\right)\right] D_{x}^{m}
$$

into

5 Although, strictly speaking, this present section is not covered by the title of the article, its content is closely related, and it should be included here for a fuller picture of the value of (1c) for nonorthogonal series. 


$$
P_{n}\left(x_{0}^{\prime}\right)+\sum_{m=1}^{n}\left[\prod_{i=0}^{m-1}\left(x-x_{i}^{\prime}\right)\right] D_{x^{\prime}}^{m},
$$

and $\Sigma_{m=0}^{n} a_{m} T_{m}^{(a, b)}$ into $\Sigma_{m=0}^{n} A_{m} T_{m}^{\left(a^{\prime}, b^{\prime}\right)}$. Furthermore, (1c) is applicable to these three frequently occurring operations:

1) Given the divided differences $D_{x}^{m}$, to find the advancing or central differences for a prescribed tabular interval $h .{ }^{6}$ We find the divided difference series in terms of the variable $p$ by replacing $D_{x}^{m}$ by $D_{p}^{m}=h^{m} D_{x}^{m}, x$ by $p$, and $x_{i}$ by $p_{i}=\left(x_{i}-x_{0}\right) / h$ before applying (1c). ${ }^{7}$

2) Conversion of an equal-interval interpolation series into a divided difference series. $^{8}$ We first convert the former into a divided difference series in the variable $p$, based upon $p_{i}$ in 1$)$, namely, $P_{n}\left(x_{0}\right)+\Sigma_{m=1}^{n}\left[\Pi_{i=0}^{m-1}\left(p-p_{i}\right)\right] D_{p}^{m}$, from which we obtain $D_{x}^{m}=D_{p}^{m} / h^{m}$.

3) Obtaining the new differences after changing the tabular interval $h$ to $h_{1}$ in any equal-interval interpolation formula (particularly, in subtabulation to a smaller interval). ${ }^{8}$ Writing the same interpolation polynomial for different intervals as

$$
P_{n}\left(x_{0}+p h\right)=\sum_{m=0}^{n} a_{m} q_{m}(p) \text { and } P_{n}\left(x_{0}+P h_{1}\right)=\sum_{m=0}^{n} A_{m} q_{m}(P)
$$

from $P=\left(h / h_{1}\right) p$ and

$$
q_{m+1}(P)+[a(m)+b(m) P] q_{m}(P)+c(m) q_{m-1}(P)=0, \quad m=0(1) n-1,
$$

noting that $q_{m}(P)$ is also $Q_{m}(p)$ in $(1 \mathrm{~b})$, we find that

$$
A(m)=a(m), \quad B(m)=\left(h / h_{1}\right) b(m) \quad \text { and } \quad C(m)=c(m), \quad m=0(1) n-1 .
$$

Similar reasoning applies in the slightly more involved case where there is both change of interval and conversion to a formula that does not have the same kind of differences (e.g., from Gregory-Newton at interval $h$ to Stirling at interval $h_{1}$ ). ${ }^{8}$ We then have $P_{n}\left(x_{0}+P h_{1}\right)=\Sigma_{m=0}^{n} A_{m} Q_{m}(P)=\Sigma_{m=0}^{n} A_{m} \bar{Q}_{m}(p)$, and from

$$
Q_{m+1}(P)+(A(m)+B(m) P) Q_{m}(P)+C(m) Q_{m-1}(P)=0, \quad m=0(1) n-1,
$$

or

(38) $\bar{Q}_{m+1}(p)+\left(A(m)+\left(h / h_{1}\right) B(m) p\right) \bar{Q}_{m}(p)+C(m) \bar{Q}_{m-1}(p)=0, \quad m=0(1) n-1$,

it is clear that we must replace only $B(m)$ by $\left(h / h_{1}\right) B(m)$ in $(1 \mathrm{c}){ }^{8}$

For another and somewhat different application of (1c), we note that it may be useful in deriving Chebyshev series approximations to solutions of difference equations, on the assumption of a polynomial approximation to the solution, whose differences of

6 The discussion here is restricted to the more usual case where $x_{0}$ is the same for both series.

7 The use of (1c) might not be always recommended for 1) and 3), save for checking or where it happens to be a more stable method. The reason is that it appears easier, in some cases, to compute $P_{n}(x)$ at the newly located equally-spaced points $x_{i}$ and then to obtain the differences, there being no divisions for 1 ) and divisions by small factorials for 3 ).

8 (See footnote 6 above.) 
some special kind, and at some particular point, are generated recursively from the equation.

Application of (1c) to Several Variables. In conclusion, it should be also noted that (1c) has applications when the $a_{m}^{(k)}$ are vector, instead of scalar quantities.

One such instance occurs in the conversion of interpolation series to Chebyshev series when we wish to have a general formula for the coefficients in the Chebyshev series in terms of the differences in the interpolation series, instead of just the numerical values of the Chebyshev coefficients corresponding to numerical values of the differences. 9 This is a practical problem when the conversions are for many sets of numerical values of the differences, and a general formula involving no divisions and fewer operations would require less work than the repeated employment of (1c) for each set of numerical differences. To obtain such general formulas, we replace in (1c) the numbers or scalars $a_{m}, m=0(1) n$, and $a_{m}^{(k)}, m=0(1) k, k=0(1) n$, by vectors whose components are numerical coefficients of the various order differences.

Another instance where vectors are employed for $a_{m}$ and $a_{m}^{(k)}$ is in the conversion of interpolation series in two variables, say $p$ and $q$, in terms of differences of mixed order and type involving both variables, expressible as

$$
\sum_{m^{\prime}=0}^{n^{\prime}} \sum_{m=0}^{n} a_{m, m^{\prime}} q_{m}(p) \bar{q}_{m^{\prime}}(q),
$$

into a double Chebyshev series of the form

$$
\sum_{m^{\prime}=0}^{n^{\prime}} \sum_{m=0}^{n} A_{m, m^{\prime}} T_{m}^{(a, b)}(p) T_{m^{\prime}}^{\left(a^{\prime}, b^{\prime}\right)}(q) .
$$

We apply (1c) first in the $q$-direction, performing $n+1$ parallel operations, for every vector $a_{m^{\prime}}^{(k)}, m^{\prime}=0(1) k, k=0(1) n^{\prime}$, having $n+1$ components which are the continually updated coefficients of $q_{m}(p), m=0(1) n$, obtaining an expression of the form

$$
\sum_{m^{\prime}=0}^{n^{\prime}}\left(\sum_{m=0}^{n} b_{m, m^{\prime}} q_{m}(p)\right) T_{m^{\prime}}^{\left(a^{\prime}, b^{\prime}\right)}(q) \text {. }
$$

We then apply (1c) in the $p$-direction, in $n^{\prime}+1$ parallel operations to each of $\sum_{m=0}^{n} b_{m, m^{\prime}} q_{m}(p), m^{\prime}=0(1) n^{\prime}$, obtaining finally $A_{m, m^{\prime}}$ above.

For more than two variables, the application of (1c) is similar. Thus for three variables, the $a_{m}^{(k)}$ in (1c) would not be vectors, but two-dimensional matrices. This would occur in the conversion of a difference formula in two variables into a double Chebyshev series whose coefficients are expressed in terms of the differences.

941 Washington Avenue

Brooklyn, New York 11225

1. J. C. P. MILLER, "Two numerical applications of Chebyshev polynomials," Proc. Roy. Soc. Edinburgh Sect. A, v. 62, 1946, pp. 204-210. MR 8, 172.

2. L. M. MILNE-THOMSON, Calculus of Finite Differences, Macmillan, London, 1933, pp. $1-7,55-70$.

3. H. E. SALZER, "A recurrence scheme for converting from one orthogonal expansion into another," Comm. $A C M$, v. 16, 1973, pp. 705-707.

9 Cf. [1], which gives the first ten Chebyshev coefficients as functions of the central differences in the Stirling and Bessel formulas through the twelfth degree, for $p$ in $[-1 / 2,1 / 2]$ and $[0,1]$, respectively. 